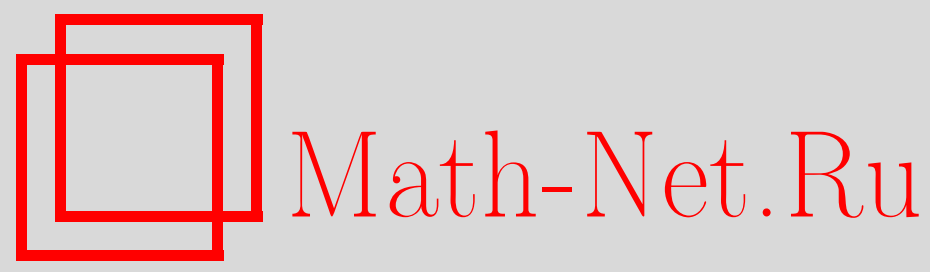

Е. А. Карацуба, Быстрое вычисление константы Каталана через приближения, полученные преобразованиями типа куммеровских, Дискрет. матем., 2013, том 25, выпуск $4,74-87$

DOI: https://doi.org/10.4213/dm1259

Использование Общероссийского математического портала Math-Net.Ru подразумевает, что вы прочитали и согласны с пользовательским соглашением http://www . mathnet.ru/rus/agreement

Параметры загрузки:

IP : 18.234 .197 .8

26 апреля 2023 г., 15:55:52

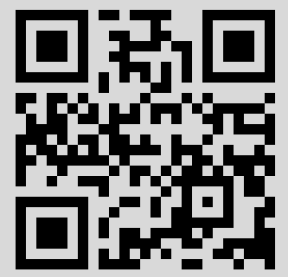




\title{
Быстрое вычисление константы Каталана через приближения, полученные преобразованиями типа куммеровских
}

\author{
() 2013 г. Е. А. Карацуба
}

Построено новое быстрое приближение константы Каталана, позволяющее вычислить эту константу со сложностью, близкой к оптимальной

Работа выполнена при поддержке РФФИ (грант № 13-01-00657).

\section{1. Введение}

Постоянная Каталана определяется соотношением

$$
G=\sum_{n=1}^{\infty} \frac{(-1)^{n-1}}{(2 n-1)^{2}}=1-\frac{1}{3^{2}}+\frac{1}{5^{2}}-\frac{1}{7^{2}}+\ldots
$$

Ряд (1) сходится медленно и не пригоден для эффективного вычисления константы $G$. Ранее (см. [1]) для быстрого вычисления $G$ использовались формулы Рамануджана (см. [2], стр. 264, стр. 289, см. также [3]), позволяющие выразить эту константу через быстро сходящиеся ряды $g_{1}$ и $g_{2}$

$$
\begin{aligned}
& g_{1}=\sum_{j=0}^{\infty} \frac{(j !)^{2}}{(2 j) !(2 j+1)^{2}}=\frac{8}{3} G-\frac{\pi}{3} \log (2+\sqrt{3}), \\
& g_{2}=\sum_{j=0}^{\infty} \frac{(2 j) !}{2^{3 j}(j !)^{2}(2 j+1)^{2}}=\frac{\pi}{4 \sqrt{2}} \log 2+\frac{1}{\sqrt{2}} G .
\end{aligned}
$$

Построенный автором метод Быстрого Вычисления Е-функций (БВЕ, см. [4]-[11], см. также [1]) даёт возможность просуммировать ряды $g_{1}$ и $g_{2}$ со сложностью, близкой к оптимальной (см. [1]). С учётом того, что с той же сложностью посредством БВЕ можно вычислить и классическую константу $\pi$ (см. [4]), а элементарные алгебраические функции можно быстро вычислить методом Ньютона, отсюда следует, что константа Каталана вычисляется по формулам Рамануджана (4), (5) с суммарной сложностью, близкой к оптимальной. Других способов быстрого вычисления константы Каталана известно не было.

Однако оказалось, что для константы Каталана можно построить быстрые приближения, в которых не будут участвовать другие классические константы, а Каталанова константа будет приближаться последовательностью рациональных чисел. 
В настоящей статье такие приближения будут построены с помощью преобразований типа куммеровских (см. [12], [13], см. также [14]) и потому будут называться куммеровскими приближениями.

Заметим, что само понятие быстрое вычисление (быстрый алгоритм) в ходе развития теории быстрых вычислений претерпевало изменения. Быстрое вычисление определяется функцией битовой сложности вычисления. Первые задачи об оценке этой функции были поставлены А.Н. Колмогоровым во второй половине 1950-х годов (см. [15]). С момента появления алгоритма умножения А. А. Карацубы в 1960 г. (см. [15], [16], [17]), под “быстрым вычислением" или вычислением с помощью "быстрого алгоритма" понимается вычисление функции, операции и т.д., битовая сложность которого по порядку ниже, чем битовая сложность вычисления этой же функции, операции и т.д. посредством алгоритмов, существовавших ранее. Ниже будет дано современное определение "быстрого алгоритма". Любое "быстрое вычисление" состоит из нескольких этапов. Первым из них является построение быстрого приближения к вычисляемому значению. Затем на основе этого приближения строится быстрый алгоритм. Поскольку с помощью БВЕ можно быстро вычислять любые ряды вида

$$
\begin{aligned}
& f_{1}=f_{1}(z)=\sum_{j=0}^{\infty} \frac{a(j)}{b(j)} z^{j}, \\
& f_{2}=f_{2}(z)=\sum_{j=0}^{\infty} \frac{a(j)}{b(j)} \frac{z^{j}}{j !},
\end{aligned}
$$

если $a(j), b(j)$ являются такими целыми числами, что $|a(j)|+|b(j)| \leqslant(C \cdot j)^{K},|z|<1$, где $K$ и $C$ - константы, а $z$ есть алгебраическое число, то, отыскав приближения $G$ вида (4), (5), мы можем получить разные БВЕ-алгоритмы вычисления постоянной Каталана.

Попытки отыскать такие приближения были и осуществлялись, в том числе, с помощью так называемой экспериментальной математики, т.е. программным путём, без доказательства справедливости полученных представлений для $G$ (о надеждах авторов, что найденная таким образом формула для постоянной Каталана верна, см. $[15])$.

С другой стороны, недавно Вальтер Ван Асшхе сообщил автору, что в библиотеке университета Лёйвена в ноябре 2013 им найден манускрипт Каталана, ранее не переводившийся на другие языки (см. [16]), где выведена формула, быстро приближающая $G$ последовательностью рациональных чисел. Отметим, что это приближение Каталана отличается от приближения, полученного в настоящей статье: Каталан находит представление $G$ в виде арифметической суммы двух быстросходящихся знакопеременных рядов, тогда как в настоящей статье построено представление $G$ в виде суммы двух быстросходящихся знакопостоянных рядов. Обе формулы: формула, доказанная Каталаном, и формула, доказанная в настоящей статье, применимы для быстрого вычисления $G$ посредством БВЕ со сложностью, близкой к оптимальной. Доказательств для других быстрых приближений константы Каталана последовательностью рациональных чисел пока не найдено. Автор благодарит Вальтера Ван Асшхе за любезное сообщение о манускрипте Каталана и содержащейся в нём формуле Каталана. 


\section{2. Получение быстрого приближения константы Ка- талана посредством преобразований типа кумме- ровских}

Здесь и далее считаем, что

$$
\begin{gathered}
n !=1 \cdot 2 \cdot 3 \ldots n, \quad(2 n-1) ! !=1 \cdot 3 \cdot 5 \ldots(2 n-1), \quad(2 n-3) ! !=1 \cdot 3 \cdot 5 \ldots(2 n-3), \\
(4 n-1) ! ! ! !=1 \cdot 3 \cdot 7 \ldots(4 n-1), \quad(4 n-3) ! ! ! !=1 \cdot 5 \cdot 9 \ldots(4 n-3),
\end{gathered}
$$

$n !=1$, если $n=0 ;(2 n-3) ! !=1$, если $n=1$.

Запишем (1) в виде

$$
\begin{gathered}
G=G_{2}-G_{1}, \\
G_{1}=\sum_{n=1}^{\infty} \frac{1}{(4 n-1)^{2}}, \\
G_{2}=\sum_{n=1}^{\infty} \frac{1}{(4 n-3)^{2}},
\end{gathered}
$$

Теорема 1. Имеет место следующее куммеровское приближение постоянной Каталана $G$

$$
G=\frac{1}{4} \sum_{n=1}^{\infty} \frac{8^{n-1}((n-1) !)^{3}}{(2 n-1) ! !}\left(\frac{6 n-3}{((4 n-3) ! ! ! !)^{2}}-\frac{6 n-1}{((4 n-1) ! ! ! !)^{2}}\right)
$$

Доказательство. Обозначим через $\tilde{S}(p)$ сумму ряда

$$
\tilde{S}(p)=\sum_{k=1}^{\infty} \frac{1}{(4 k-1)^{2}(4 k+3)^{2}(4 k+7)^{2} \ldots(4 k+4 p-5)^{2}} .
$$

Преобразуем (10), воспользовавшись схемой из [14]. Определим $\tilde{D}_{k}(p)$ как разность

$$
\begin{gathered}
\tilde{D}_{k}(p)=\frac{4 k-1+x}{(4 k-1)^{2}(4 k+3)^{2} \ldots(4 k+4 p-5)^{2}}-\frac{4 k+3+x}{(4 k+3)^{2}(4 k+7)^{2} \ldots(4 k+4 p-1)^{2}} \\
=\frac{(4 k-1+x)(4 k+4 p-1)^{2}-(4 k+3+x)(4 k-1)^{2}}{(4 k-1)^{2}(4 k+3)^{2}(4 k+7)^{2} \ldots(4 k+4 p-5)^{2}(4 k+4 p-1)^{2}}
\end{gathered}
$$

при некотором, определяемом в дальнейшем, параметре $x$. Упростим числитель дроби (11)

$$
\tilde{D}_{k}(p)=\frac{4(2 p-1)(4 k-1)^{2}+(4 k-1)\left((4 p)^{2}+8 p x\right)+x(4 p)^{2}}{(4 k-1)^{2}(4 k+3)^{2}(4 k+7)^{2} \ldots(4 k+4 p-5)^{2}(4 k+4 p-1)^{2}}
$$

и при $k \rightarrow \infty$

$$
\frac{1}{4(2 p-1)} \tilde{D}_{k}(p)=\frac{1}{4(2 p-1)}\left(\frac{4 k-1+x}{(4 k-1)^{2}(4 k+3)^{2} \ldots(4 k+4 p-5)^{2}}-\right.
$$




$$
\left.-\frac{4 k+3+x}{(4 k+3)^{2}(4 k+7)^{2} \ldots(4 k+4 p-1)^{2}}\right) \sim \frac{1}{(4 k-1)^{2}(4 k+3)^{2} \ldots(4 k+4 p-5)^{2}} .
$$

Если заменить члены ряда (10) этими эквивалентными им разностями из (13), то получим новый ряд

$$
\tilde{D}(p)=\frac{1}{4(2 p-1)} \sum_{k=1}^{\infty} \tilde{D}_{k}(p)
$$

с частной суммой ряда

$$
\begin{gathered}
\tilde{D}_{K}(p)=\frac{1}{4(2 p-1)} \sum_{k=1}^{K} \tilde{D}_{k}(p)= \\
=\frac{1}{4(2 p-1)}\left(\frac{3+x}{3^{2} \cdot 7^{2} \cdot \ldots \cdot(4 p-1)^{2}}-\frac{7+x}{7^{2} \cdot 11^{2} \cdot \ldots \cdot(4 p+3)^{2}}+\right. \\
\left.+\frac{7+x}{7^{2} \cdot 11^{2} \cdot \ldots \cdot(4 p+3)^{2}}-\ldots-\frac{4 K+3+x}{(4 K+3)^{2} \ldots(4 K+4 p-1)^{2}}\right)
\end{gathered}
$$

и суммой ряда

$$
\tilde{D}(p)=\frac{1}{4(2 p-1)} \frac{3+x}{3^{2} \cdot 7^{2} \cdot \ldots \cdot(4 p-1)^{2}} .
$$

Обозначим через $\tilde{r}(p)$ разность

$$
\tilde{r}(p)=\tilde{S}(p)-\tilde{D}(p)=\sum_{k=1}^{\infty} \tilde{\rho}_{k}(p)
$$

где с учётом (12), (14)

$$
\begin{aligned}
\tilde{\rho}_{k}(p)= & \frac{(4 k+4 p-1)^{2}-(4 k-1)^{2}-(4 k-1) \frac{(4 p)^{2}+8 p x}{4(2 p-1)}-\frac{x(4 p)^{2}}{4(2 p-1)}}{(4 k-1)^{2}(4 k+3)^{2} \ldots(4 k+4 p-5)^{2}(4 k+4 p-1)^{2}}= \\
& =\frac{(4 k-1)\left(8 p-\frac{8 p x+(4 p)^{2}}{4(2 p-1)}\right)+(4 p)^{2}\left(1-\frac{x}{4(2 p-1)}\right)}{(4 k-1)^{2}(4 k+3)^{2} \ldots(4 k+4 p-5)^{2}(4 k+4 p-1)^{2}} .
\end{aligned}
$$

Выберем теперь произвольный параметр $x$, положив

$$
x=6 p-4 .
$$

В этом случае в числителе дроби (17) выражение, стоящее в скобках при множителе $(4 k-1)$, обращается в ноль, и в результате получаем

$$
\tilde{\rho}_{k}(p)=\frac{8 p^{3}}{2 p-1} \frac{1}{(4 k-1)^{2}(4 k+3)^{2} \ldots(4 k+4 p-5)^{2}(4 k+4 p-1)^{2}} .
$$

Из (14)-(19) находим

$$
\tilde{S}(p)=\tilde{D}(p)+\sum_{k=1}^{\infty} \frac{8 p^{3}}{2 p-1} \frac{1}{(4 k-1)^{2}(4 k+3)^{2} \ldots(4 k+4 p-5)^{2}(4 k+4 p-1)^{2}}=
$$




$$
\begin{gathered}
=\frac{1}{4(2 p-1)} \frac{3+6 p-4}{3^{2} \cdot 7^{2} \cdot \ldots \cdot(4 p-1)^{2}}+\frac{8 p^{3}}{2 p-1} \tilde{S}(p+1)= \\
=\frac{6 p-1}{4(2 p-1)((4 p-1) ! ! ! !)^{2}}+\frac{8 p^{3}}{2 p-1} \tilde{S}(p+1) .
\end{gathered}
$$

Из и (10) и (20) вычисляем

$$
\begin{gathered}
\tilde{S}(1)=\sum_{k=1}^{\infty} \frac{1}{(4 k-1)^{2}}=\frac{5}{4 \cdot 3^{2}}+8 \tilde{S}(2)=\frac{5}{36}+8 \tilde{S}(2), \\
8 \tilde{S}(2)=8 \frac{11}{4 \cdot 3^{2} \cdot 7^{2}}+8 \cdot \frac{8^{2}}{3} \tilde{S}(3)=\frac{88}{5292}+\frac{8^{3}}{3} \tilde{S}(3) .
\end{gathered}
$$

И так далее. Отсюда находим следующую рекуррентную формулу

$$
\frac{8^{p-1}((p-1) !)^{3}}{(2 p-3) ! !} \tilde{S}(p)=\frac{8^{p-1}(6 p-1)((p-1) !)^{3}}{4(2 p-1) ! !((4 p-1) ! ! ! !)^{2}}+\frac{8^{p}(p !)^{3}}{(2 p-1) ! !} \tilde{S}(p+1) .
$$

Таким образом, из (19)-(21) имеем

$$
\begin{aligned}
\sum_{k=1}^{\infty} \frac{1}{(4 k-1)^{2}} & =\frac{5}{36}+\frac{88}{5292}+\frac{2176}{800415}+\ldots+\frac{8^{m-1}(6 m-1)((m-1) !)^{3}}{4(2 m-1) ! !((4 m-1) ! ! ! !)^{2}}+\ldots+ \\
& +\frac{8^{p}(p !)^{3}}{(2 p-1) ! !} \sum_{k=1}^{\infty} \frac{1}{(4 k-1)^{2}(4 k+3)^{2} \ldots(4 k+4 p-1)^{2}}
\end{aligned}
$$

Последний член в (22)

$$
\begin{aligned}
\tilde{R}(p)= & \frac{8^{p}(p !)^{3}}{(2 p-1) ! !} \sum_{k=1}^{\infty} \frac{1}{(4 k-1)^{2}(4 k+3)^{2} \ldots(4 k+4 p-1)^{2}} \leqslant \frac{2^{3 p}(p !)^{3}}{(2 p-1) ! !} \frac{2}{((4 p+3) ! ! ! !)^{2}} \leqslant \\
& \leqslant \frac{\left.2 \cdot 2^{3 p}(\Gamma(p+1))^{3}\right) \Gamma\left(\frac{1}{2}\right)\left(\Gamma\left(\frac{3}{4}\right)\right)^{2}}{2^{p} \Gamma\left(p+\frac{1}{2}\right)\left(\Gamma\left(p+1+\frac{3}{4}\right) \cdot 4^{p+1}\right)^{2}} \leqslant \frac{2^{3 p+1} \Gamma\left(\frac{1}{2}\right)\left(\Gamma\left(\frac{3}{4}\right)\right)^{2} p \Gamma(p)}{2^{5 p+4} \Gamma\left(p+\frac{1}{2}\right)}<\frac{p}{2^{2 p}}
\end{aligned}
$$

стремится к нулю при $p \rightarrow \infty$. Здесь учтено, что $\Gamma\left(\frac{1}{2}\right)=\sqrt{\pi}=1.77 \ldots<2, \Gamma\left(\frac{3}{4}\right)=$ $1.22 \ldots<2$, а также, что

$$
\begin{aligned}
& \sum_{k=1}^{\infty} \frac{1}{(4 k-1)^{2}(4 k+3)^{2} \ldots(4 k+4 p-1)^{2}}=\frac{1}{((4 p+3) ! ! ! !)^{2}}\left(1+\frac{3^{2}}{(4 p+7)^{2}}+\right. \\
& \left.\quad+\frac{3^{2} \cdot 7^{2}}{(4 p+7)^{2}(4 p+11)^{2}}+\ldots\right) \leqslant \frac{1}{((4 p+3) ! ! ! !)^{2}}\left(1+\frac{3^{2}}{4^{2}(p+1+3 / 4)^{2}}+\right. \\
& \left.+\frac{3^{2}}{4^{2}(p+2+3 / 4)^{2}}+\ldots\right) \leqslant \frac{1}{((4 p+3) ! ! ! !)^{2}}\left(1+\left(\frac{3}{4}\right)^{2} \zeta(2)\right)<\frac{2}{((4 p+3) ! ! ! !)^{2}} .
\end{aligned}
$$

Отсюда окончательно получаем

$$
G_{1}=\frac{1}{4} \sum_{n=1}^{\infty} \frac{8^{n-1}(6 n-1)((n-1) !)^{3}}{(2 n-1) ! !((4 n-1) ! ! ! !)^{2}} .
$$


Куммеровское приближение для константы $G_{2}$ из (8) находится аналогично. При этом вместо суммы $\tilde{S}(p)$ рассматривается сумма ряда $\tilde{\tilde{S}}(p)$,

$$
\tilde{\tilde{S}}(p)=\sum_{k=1}^{\infty} \frac{1}{(4 k-3)^{2}(4 k+1)^{2}(4 k+5)^{2} \ldots(4 k+4 p-7)^{2}} .
$$

Рекуррентная формула для $\tilde{\widetilde{S}}(p)$ будет иметь вид

$$
\frac{8^{p-1}((p-1) !)^{3}}{(2 p-3) ! !} \tilde{\tilde{S}}(p)=\frac{8^{p-1}(6 p-3)((p-1) !)^{3}}{4(2 p-1) ! !((4 p-3) ! ! ! !)^{2}}+\frac{8^{p}(p !)^{3}}{(2 p-1) ! !} \tilde{\widetilde{S}}(p+1) .
$$

Проводя вычисления по формуле (25) последовательно получим

$$
\begin{gathered}
\sum_{k=1}^{\infty} \frac{1}{(4 k-3)^{2}}=\frac{3}{4}+\frac{6}{25}+\frac{128}{2025}+\ldots+\frac{8^{m-1}(6 m-3)((m-1) !)^{3}}{4(2 m-1) ! !((4 m-3) ! ! ! !)^{2}}+\ldots+ \\
+\frac{8^{p}(p !)^{3}}{(2 p-1) ! !} \sum_{k=1}^{\infty} \frac{1}{(4 k-3)^{2}(4 k+1)^{2} \ldots(4 k+4 p-3)^{2}} .
\end{gathered}
$$

Так как последний член в (26) стремится к нулю при $p \rightarrow \infty$, то окончательно для постоянной $G_{2}$ получаем

$$
G_{2}=\frac{1}{4} \sum_{n=1}^{\infty} \frac{8^{n-1}(6 n-3)((n-1) !)^{3}}{(2 n-1) ! !((4 n-3) ! ! ! !)^{2}}=\frac{3}{4} \sum_{n=1}^{\infty} \frac{8^{n-1}((n-1) !)^{3}}{(2 n-3) ! !((4 n-3) ! ! ! !)^{2}} .
$$

Из (6)-(8) и (23), (25) получаем куммеровское приближение постоянной Каталана $G(9)$.

Замечание 1. Поскольку (чем мы уже воспользовались при оценке остаточного члена в формуле (22))

$$
(2 n-1) ! !=\frac{\Gamma\left(n+\frac{1}{2}\right) 2^{n}}{\Gamma\left(\frac{1}{2}\right)}, \quad(4 n-1) ! ! ! !=\frac{\Gamma\left(n+\frac{3}{4}\right) 4^{n}}{\Gamma\left(\frac{3}{4}\right)}, \quad(4 n-3) ! ! ! !=\frac{\Gamma\left(n+\frac{1}{4}\right) 4^{n}}{\Gamma\left(\frac{1}{4}\right)},
$$

куммеровское приближение константы Каталана (9) можно записать в виде

$$
G=\sum_{n=1}^{\infty} \frac{(\Gamma(n))^{3} \Gamma\left(\frac{1}{2}\right)}{2^{2 n+5} \Gamma\left(n+\frac{1}{2}\right)}\left(\frac{\Gamma^{2}\left(\frac{1}{4}\right)}{\Gamma^{2}\left(n+\frac{1}{4}\right)}(6 n-3)-\frac{\Gamma^{2}\left(\frac{3}{4}\right)}{\Gamma^{2}\left(n+\frac{3}{4}\right)}(6 n-1)\right),
$$

или более компактно, через бэта-функции

$$
G=\sum_{n=1}^{\infty} \frac{B\left(n, \frac{1}{2}\right)}{2^{2 n+5}}\left(B^{2}\left(n, \frac{1}{4}\right)(6 n-3)-B^{2}\left(n, \frac{3}{4}\right)(6 n-1)\right) .
$$

Замечание 2. На практике можно несколько улучшить эффективность вычисления постоянной $G$, подсчитав отдельно сумму $k$ первых дробей из (1) и применяя преобразование типа куммеровского к остальному ряду. Таким образом получим 


$$
\begin{gathered}
G=\sum_{n=1}^{\infty} \frac{B\left(n, \frac{1}{2}\right)}{2^{2 n+5}}\left(B^{2}\left(n, \frac{1}{4}\right)(6 n-3)-B^{2}\left(n, \frac{3}{4}\right)(6 n-1)\right)= \\
=1+\sum_{n=1}^{\infty} \frac{B\left(n, \frac{1}{2}\right)}{2^{2 n+5}}\left(B^{2}\left(n, \frac{1}{4}\right) \frac{6 n+1}{(4 n+1)^{2}}-B^{2}\left(n, \frac{3}{4}\right)(6 n-1)\right)= \\
=\frac{8}{9}+\sum_{n=1}^{\infty} \frac{B\left(n, \frac{1}{2}\right)}{2^{2 n+5}}\left(B^{2}\left(n, \frac{1}{4}\right) \frac{6 n+1}{(4 n+1)^{2}}-B^{2}\left(n, \frac{3}{4}\right) \frac{3^{2}(6 n+3)}{(4 n+3)^{2}}\right)= \\
=\frac{10016}{11025}+\sum_{n=1}^{\infty} \frac{B\left(n, \frac{1}{2}\right)}{2^{2 n+5}}\left(B^{2}\left(n, \frac{1}{4}\right) \frac{5^{2}(6 n+5)}{(4 n+1)^{2}(4 n+5)^{2}}-B^{2}\left(n, \frac{3}{4}\right) \frac{3^{2} \cdot 7^{2}(6 n+7)}{(4 n+3)^{2}(4 n+7)^{2}}\right) .
\end{gathered}
$$

И так далее. Однако, с точки зрения сложности вычисления, такие преобразования заметного эффекта не дают.

\section{3. БВЕ-алгоритм вычисления константы Каталана}

Будем считать, что числа записаны в двоичной системе счисления (для десятичной системы счисления алгоритмы те же, и все рассуждения такие же, с той разницей, что под словами "с точностью до $n$ знаков" в случае двоичной системы подразумевается точность $2^{-n}$, для десятичных же вычислений $-10^{-n}$, и это нужно учитывать в соответствующих оценках). Здесь $n-$ натуральное число, $n \rightarrow+\infty$.

Применим к вычислению постоянной Каталана метод БВЕ (см. [4]-[11], см. также [1]). Для этого просуммируем с помощью БВЕ-процесса оба ряда $G_{1}$ и $G_{2}$ по отдельности, используя их куммеровские приближения (23) и (27) соответственно. Перепишем (23) в виде

$$
G_{1}=\frac{1}{2^{5}} S
$$

где

$$
S=\sum_{k=1}^{\infty} \frac{2^{3 k}(6 k-1)((k-1) !)^{3}}{(2 k-1) ! !((4 k-1) ! ! ! !)^{2}}
$$

Сначала оценим остаток ряда (31).

$$
S=S_{r}+R_{r}
$$

где

$$
\begin{gathered}
S_{r}=\sum_{k=1}^{r} \frac{2^{3 k}(6 k-1)((k-1) !)^{3}}{(2 k-1) ! !((4 k-1) ! ! ! !)^{2}} \\
R_{r}=\sum_{k=r+1}^{\infty} \frac{2^{3 k}(6 k-1)((k-1) !)^{3}}{(2 k-1) ! !((4 k-1) ! ! ! !)^{2}}=\sum_{k=r+1}^{\infty} \frac{(6 k-1)(\Gamma(k))^{3} \Gamma^{2}\left(\frac{3}{4}\right) \Gamma\left(\frac{1}{2}\right)}{2^{2 k} \Gamma\left(k+\frac{1}{2}\right) \Gamma^{2}\left(k+\frac{3}{4}\right)} .
\end{gathered}
$$

Учитывая, что $\Gamma\left(\frac{1}{2}\right)=\sqrt{\pi}=1.77 \ldots<2, \Gamma\left(\frac{3}{4}\right)=1.22 \ldots<2$, а также что при $x \geqslant$ $r+1 \geqslant 2$, гамма-функция $\Gamma(x)$ монотонно возрастает, из (34) имеем 


$$
R_{r} \leqslant \sum_{k=r+1}^{\infty} \frac{3(6 k-1)}{2^{2 k}} \leqslant \frac{6 r+7}{2^{2 r}} .
$$

Из (35) следует, что при

$$
r=n, \quad n \geqslant 4,
$$

остаток ряда $(31) R_{n} \leqslant 2^{-n}$. Возьмём

$$
r=2^{m}, m \geqslant 1 ; \quad 2^{m-1}<n \leqslant 2^{m}
$$

членов ряда (31). Из вышеприведённых оценок следует, что для того чтобы вычислить сумму ряда (31) с точностью до $n$ знаков, достаточно вычислить сумму (33) при условии (36). Пусть $s_{r-\nu}(0), \nu=0,1,2, \ldots, r-1$, определяются равенствами

$$
s_{r-\nu}(0)=\frac{2^{3(r-\nu)}(6(r-\nu)-1)((r-\nu-1) !)^{3}}{(2(r-\nu)-1) ! !((4(r-\nu)-1) ! ! ! !)^{2}},
$$

По определению $S_{r}$ имеем

$$
S_{r}=s_{1}(0)+s_{2}(0)+\ldots+s_{r}(0) .
$$

Вычисление суммы (39) проведём за $m$ шагов БВЕ-процесса. Объединяя на каждом шаге слагаемые $S_{r}$ последовательно попарно и вынося за скобки "очевидный" общий множитель, будем вычислять на каждом шаге (до последнего) только целые значения выражений в скобках. При наличии ограничений на длину чисел (в реальных компьютерах), этот алгоритм можно модифицировать, образуя в скобках дроби и вычисляя отдельно целые числители и целые знаменатели соответствующих дробей. Здесь однако, мы предполагаем, что ограничений на количество знаков, участвующих в вычислениях чисел, нет, и наша цель лишь получить хорошую оценку суммарной сложности вычисления, когда точность $n$ растёт, $n \rightarrow+\infty$.

Таким образом, после первого шага имеем:

$$
\begin{gathered}
S_{r}=s_{1}(1)+s_{2}(1)+\ldots+s_{r_{1}}(1), \quad r_{1}=\frac{r}{2}, \\
s_{r_{1}-\nu}(1)=s_{r-2 \nu}(0)+s_{r-2 \nu-1}(0)=\frac{2^{3(r-2 \nu)}(6(r-2 \nu)-1)((r-2 \nu-1) !)^{3}}{(2(r-2 \nu)-1) ! !((4(r-2 \nu)-1) ! ! ! !)^{2}}+ \\
+\frac{2^{3(r-2 \nu-1)}(6(r-2 \nu-1)-1)((r-2 \nu-2) !)^{3}}{(2(r-2 \nu-1)-1) ! !((4(r-2 \nu-1)-1) ! ! ! !)^{2}}= \\
=\frac{2^{3(r-2 \nu-1)}((r-2 \nu-2) !)^{3}}{(2(r-2 \nu)-1) ! !((4(r-2 \nu)-1) ! ! ! !)^{2}} \alpha_{r_{1}-\nu}(1) .
\end{gathered}
$$

На первом шаге вычисляются числа $\alpha_{r_{1}-\nu}(1), \nu=0,1,2, \ldots, r_{1}-1, r_{1}=\frac{r}{2}=2^{m-1}$ :

$$
\alpha_{r_{1}-\nu}(1)=2^{3}(r-2 \nu-1)^{3}(6 r-12 \nu-1)+(2 r-4 \nu-1)(4 r-8 \nu-1)^{2}(6 r-12 \nu-7) .
$$

После второго шага имеем: 


$$
\begin{gathered}
S_{r}=s_{1}(2)+s_{2}(2)+\ldots+s_{r_{2}}(2), \quad r_{2}=\frac{r_{1}}{2}=\frac{r}{2^{2}}, \\
s_{r_{2}-\nu}(2)=s_{r_{1}-2 \nu}(1)+s_{r_{1}-2 \nu-1}(1)=\frac{2^{3(r-4 \nu-1)}((r-4 \nu-2) !)^{3}}{(2(r-4 \nu)-1) ! !((4(r-4 \nu)-1) ! ! ! !)^{2}} \alpha_{r_{2}-\nu}(2) .
\end{gathered}
$$

На втором шаге вычисляются числа $\alpha_{r_{2}-\nu}(2), \nu=0,1,2, \ldots, r_{2}-1, r_{2}=\frac{r_{1}}{2}=2^{m-2}$ :

$$
\begin{gathered}
\alpha_{r_{2}-\nu}(2)=2^{6}\left(r-2^{2} \nu-3\right)^{3}\left(r-2^{2} \nu-2\right)^{3} \alpha_{r_{1}-2 \nu}(1)+ \\
+\left(2 r-2^{3} \nu-3\right)\left(2 r-2^{3} \nu-1\right)\left(4 r-2^{4} \nu-5\right)^{2}\left(4 r-2^{4} \nu-1\right)^{2} \alpha_{r_{1}-2 \nu-1}(1) .
\end{gathered}
$$

И так далее. После $j$-го, $1<j \leqslant m$, шага получаем:

$$
\begin{gathered}
S_{r}=s_{1}(j)+s_{2}(j)+\ldots+s_{r_{j}-1}(j)+s_{r_{j}}(j), \quad r_{j}=\frac{r}{2^{j}}, \\
s_{r_{j}-\nu}(j)=\frac{2^{3\left(r-2^{j} \nu-2^{j}+1\right)}\left(\left(r-2^{j} \nu-2^{j}\right) !\right)^{3}}{\left(2\left(r-2^{j} \nu\right)-1\right) ! !\left(\left(4\left(r-2^{j} \nu\right)-1\right) ! ! ! !\right)^{2}} \alpha_{r_{j}-\nu}(j) .
\end{gathered}
$$

На $j$-м шаге вычисляются числа $\alpha_{r_{j}-\nu}(j), \nu=0,1,2, \ldots, r_{j}-1, r_{j}=\frac{r_{j-1}}{2}=\frac{r}{2^{j}}=$ $2^{m-j}$ :

$$
\begin{gathered}
\alpha_{r_{j}-\nu}(j)=2^{3 \cdot 2^{j-1}}\left(\frac{\left(r-2^{j} \nu-2^{j-1}\right) !}{\left(r-2^{j} \nu-2^{j}\right) !}\right)^{3} \alpha_{r_{j-1}-2 \nu}(j-1)+ \\
+\frac{\left(2\left(r-2^{j} \nu\right)-1\right) ! !}{\left(2\left(r-2^{j} \nu-2^{j-1}\right)-1\right) ! !}\left(\frac{\left(4\left(r-2^{j} \nu\right)-1\right) ! ! ! !}{\left(4\left(r-2^{j} \nu-2^{j-1}\right)-1\right) ! ! ! !}\right)^{2} \alpha_{r_{j-1}-2 \nu-1}(j-1) .
\end{gathered}
$$

И так далее. После $m$ шагов БВЕ-процесса имеем:

$$
S_{r}=s_{r_{m}}(m)=s_{1}(m)=\frac{2^{3} \alpha_{r_{m}}(m)}{(2 r-1) ! !((4 r-1) ! ! ! !)^{2}}, \quad r_{m}=\frac{r}{2^{m}}=1 .
$$

На последнем $m$-м шаге вычисляем одно целое число $\alpha_{r_{m}}(m)=\alpha_{1}(m)$, целые числа $(2 r-1) ! !,(4 r-1) ! ! ! !$, возводим последнее число в квадрат и вычисляем произведение $(2 r-1) ! !((4 r-1) ! ! ! !)^{2}$, а затем производим одно деление с точностью до $n$ знаков целого числа $8 \alpha_{r_{m}}(m)$ на целое число $(2 r-1) ! !((4 r-1) ! ! ! !)^{2}$. Результатом будет число, равное (см. (30)-(32)) значению суммы $S$ с точностью до $n$ знаков. Поделив его на $2^{5}$ получим значение $G_{1}$ с точностью до $n+5$ знаков.

Аналогичным образом строится БВЕ-алгоритм для вычисления $G_{2}$ на основе куммеровского приближения (27). Вычисляя разность найденных значений $G_{1}$ и $G_{2}$ (см. (6)), в результате получим константу Каталана $G$ с точностью до $n$ знаков.

\section{4. Сложность вычисления константы Каталана}

Чтобы доказать, что вышеприведённый БВЕ-алгоритм является быстрым алгоритмом, нужно оценить сложность проводимых на каждом шаге вычислений и оценить суммарную сложность вычисления константы $G$ в битовых о операциях (см. [17], $[18])$. 
Напомним, что сложностью вычисления заданной функции $f(x)$ в точке $x=x_{0}$ (с точностью до $n$ знаков) называется количество битовых операций $s_{f}(n)$, достаточное для вычисления функции $f(x)$ в точке $x=x_{0}$ с точностью до $n$ знаков посредством данного алгоритма. При этом функция сложности умножения (двух $n$-значных чисел) имеет специальное обозначение $M(n)$.

Первым быстрым алгоритмом (не известно ни одной более ранней публикации с каким-либо быстрым методом или алгоритмом) явился алгоритм быстрого умножения, найденный А. А. Карацубой (см. [17], [18], [19]) в 1960 г., сложность которого

$$
M(n)=O\left(n^{\alpha}\right), \quad \alpha=\log _{2} 3=1,5849 \ldots
$$

Впоследствии идея А. А. Карацубы была обобщена, использована напрямую или в модифицированном виде под многими названиями (такими, например, как "divide and conquer" и "binary splitting") и во многих алгоритмах, самыми известными из которых, по-видимому, являются Быстрое Преобразование Фурье и алгоритмы умножения чисел и матриц.

Первым обобщением умножения А. А. Карацубы, был алгоритм умножения А. Л. Тоома (см. [20]). Следующим и, вероятно, вторым обобщением явился алгоритм самого А. А. Карацубы со сложностью

$$
M(n)=O(n \log n \log \log n \log \log \log n \ldots) .
$$

Однако статья с этим алгоритмом не была опубликована (см. [21]). Затем А. Шёнхаге и Ф. Штрассен разработали алгоритм (см. [22]) со сложностью вычисления

$$
M(n)=O(n \log n \log \log n),
$$

который долгое время оставался наибыстрейшим (о новом 2007 года алгоритме умножения М. Фюрера см. [23]). Далее при учёте сложности умножения будем использовать оценку Шёнхаге-Штрассена (46) (можно пользоваться также оценкой (45) или оценкой Фюрера из [23], однако обе они гораздо более громоздки, и потому предпочтение отдано (46)).

Таким образом, будем называть “быстрым" такой алгоритм вычисления функции $f=f(x)$, что для этого алгоритма

$$
s_{f}(n)=O\left(M(n) \log ^{c} n\right),
$$

где $c$ есть константа, и для $M(n)$ справедлива оценка Шёнхаге-Штрассена. Ясно, что для любого алгоритма и любой функции $f$, выполняется неравентство:

$$
s_{f}(n)>n \text {. }
$$

(Заметим, что только запись числа с точностью до $n$ знаков требует не меньше, чем $n+1$ операций.) Следовательно, для быстрого алгоритма

$$
n<s_{f}(n)<c_{1} n \log ^{c+1} n \log \log n<n^{1+\varepsilon},
$$

для любого $\varepsilon>0$ и $n>n_{1}(\varepsilon)$. Тем самым, быстрым алгоритмам (быстрым вычислениям) соответствует правильный порядок оценки сверху $s_{f}(n)$ по $n$ при $n \rightarrow \infty$. Так, построенный автором БВЕ-процесс суммирования рядов (4), (5) имеет соответственно сложность (см., например, [1], [4])

$$
s_{f_{1}}(n)=O\left(M(n) \log ^{2} n\right)=O\left(n \log ^{3} n \log \log n\right)=O\left(n^{1+\varepsilon}\right),
$$




$$
s_{f_{2}}(n)=O(M(n) \log n)=O\left(n \log ^{2} n \log \log n\right)=O\left(n^{1+\varepsilon}\right),
$$

для любого $\varepsilon>0$ и $n>n_{1}(\varepsilon)$, и является быстрым процессом.

Воспользовавшись БВЕ-алгоритмом §3, можно доказать следующее утверждение

Теорема 2. Для сложности $s_{G}(n)$ вычисления постоянной Каталана $G$ справедлива оценка

$$
s_{G}(n)=\left(O\left(M(n) \log ^{2} n\right)=O\left(n \log ^{3} n \log \log n\right) .\right.
$$

Доказательство. Оценим сложность вычисления константы $G_{1}$.

Подсчитаем количество битовых операций, достаточное для вычисления суммы $S$ с точностью $2^{-n}$. Для этого мы производим вычисление суммы $S_{r}, r=2^{m} ; 2^{m-1}<$ $n \leqslant 2^{m}$, за $m$ шагов БВЕ-процесса, описанного в параграфе 3 .

Сначала, пользуясь формулой (43), подсчитаем количество операций, достаточное для вычисления на $j+1$-ом шаге, $j+1 \leqslant m$, чисел $\alpha_{r_{j+1}-\nu}(j+1)$, $\nu=0,1,2, \ldots, r_{j+1}-1, r_{j+1}=\frac{r}{2^{j+1}}$, предполагая при этом, что числа $\alpha_{\mu}(j)$, $j=1,2, \ldots, m-1$, уже вычислены. Для этого, прежде всего, оценим сверху разрядность чисел, с которыми проводятся вычисления на $(j+1)$-м шаге.

Пусть

$$
\alpha(j)=\max _{\mu} \alpha_{\mu}(j)
$$

Так как

$$
\begin{gathered}
\left(\frac{\left(r-2^{j+1} \nu-2^{j}\right) !}{\left(r-2^{j+1} \nu-2^{j+1}\right) !}\right)^{3} \leqslant\left(r^{2^{j}}\right)^{3}, \\
\frac{\left(2 r-2^{j+2} \nu-1\right) ! !}{\left(2 r-2^{j+2} \nu-2^{j+1}-1\right) ! !} \leqslant(2 r)^{2^{j}}, \\
\left(\frac{\left(4 r-2^{j+3} \nu-1\right) ! ! ! !}{\left(4 r-2^{j+3} \nu-2^{j+2}-1\right) ! ! ! !}\right)^{2} \leqslant\left((4 r)^{2^{j}}\right)^{2},
\end{gathered}
$$

то из (43) имеем

$$
\alpha(j+1) \leqslant \alpha(j)\left((2 r)^{3 \cdot 2^{j}}+(2 r)^{2^{j}}(4 r)^{2^{j+1}}\right) \leqslant(4 r)^{2^{j+2}} .
$$

Отсюда находим

$$
\frac{\alpha(j+1)}{\alpha(j)} \leqslant(4 r)^{2^{j+2}}, \quad \frac{\alpha(j+1)}{\alpha(1)} \leqslant(4 r)^{2^{j+3}-2^{3}} .
$$

Поскольку $\alpha(1) \leqslant 240 r^{4}$ в силу (42), из (48) получаем, что

$$
\alpha(j+1) \leqslant(4 r)^{2^{j+3}} .
$$

Для вычисления произведений

$$
\begin{gathered}
\frac{\left(r-2^{j+1} \nu-2^{j}\right) !}{\left(r-2^{j+1} \nu-2^{j+1}\right) !}, \\
\frac{\left(2 r-2^{j+2} \nu-1\right) ! !}{\left(2 r-2^{j+2} \nu-2^{j+1}-1\right) ! !},
\end{gathered}
$$




$$
\frac{\left(4 r-2^{j+3} \nu-1\right) ! ! ! !}{\left(4 r-2^{j+3} \nu-2^{j+2}-1\right) ! ! ! !}
$$

в [4] построен БВЕ-процесс, позволяющий вычислить первое из произведений со сложностью

$$
O\left(\sum_{i=1}^{j} M\left(2^{i} \log r\right)\right)
$$

а второе и третье произведения со сложностями

$$
O\left(\sum_{i=1}^{j} M\left(2^{i} \log 2 r\right)\right) \text { и } O\left(\sum_{i=1}^{j} M\left(2^{i} \log 4 r\right)\right)
$$

соответственно. Чтобы возвести $2^{j} \log r$-значное число $\frac{\left(r-2^{j+1} \nu-2^{j}\right) !}{\left(r-2^{j+1} \nu-2^{j+1}\right) !}$ в куб, достаточно $O\left(M\left(2^{j+1} \log r\right)\right)$ операций, а чтобы возвести $2^{j} \log 4 r$-значное число $\frac{\left(4 r-2^{j+3} \nu-1\right) ! ! ! !}{\left(4 r-2^{j+3} \nu-2^{j+2}-1\right) ! ! ! !}$ в квадрат, достаточно $O\left(M\left(2^{j} \log 4 r\right)\right)$ операций. Чтобы перемножить $2^{j} \log 2 r$-значное число $\frac{\left(2 r-2^{j+2} \nu-1\right) ! !}{\left(2 r-2^{j+2} \nu-2^{j+1}-1\right) ! !}$ и $2^{j+1} \log 4 r$-значное число $\left(\frac{\left(4 r-2^{j+3} \nu-1\right) ! ! ! !}{\left(4 r-2^{j+3} \nu-2^{j+2}-1\right) ! ! ! !}\right)^{2}$, достаточно $O\left(M\left(2^{j+1} \log 4 r\right)\right)$ операций. Следовательно, для вычисления $\alpha_{r_{j+1}-\nu}(j+1)$ (см. формулу $(42)$ для $\left.j+1\right)$, достаточно $\left.O(A(j+1))\right)$ операций, где

$$
\begin{gathered}
A(j+1)= \\
=\sum_{i=1}^{j} M\left(2^{i} \log 4 r\right)+M\left(2^{j+1} \log 4 r\right)= \\
=O\left(2^{j+1} \log r(j+1+\log \log r)(\log (j+1+\log \log r))\right) .
\end{gathered}
$$

Чтобы вычислить все $\alpha_{r_{j+1}-\nu}(j+1)$, которых ровно $r_{j+1}=2^{-(j+1)} r$, достаточно

$$
O\left(r_{j+1} A(j+1)\right)
$$

операций. Тогда для вычисления $S$ достаточно

$$
\begin{gathered}
O\left(\sum_{j=1}^{m-1} r_{j+1} A(j+1)\right)= \\
=O\left(r \sum_{j=1}^{m-1} \log r(j+1+\log \log r)(\log (j+1+\log \log r))\right)= \\
=O\left(r \log ^{3} r \log \log r\right) .
\end{gathered}
$$

Количество битовых операций, достаточное для вычисления константы $G_{2}$, подсчитывается аналогично, и для сложности вычисления $G_{2}$ также справедлива оценка 
(50). Из (6), (36),(46) и (50) следует, что для вычисления постоянной Каталана $G$ с точностью до $n$ знаков достаточно

$$
O\left(n \log ^{3} n \log \log n\right)=O\left(M(n) \log ^{2} n\right)
$$

операций.

\section{Список литературы}

1. Karatsuba E. A., Fast computation of some special integrals of mathematical physics. Sci. Comput., Validated Numerics, Interval Methods (2001) 29-41.

2. Berndt B. C., Ramanujan's notebooks, 1. Springer, 1985.

3. Ramanujan S., Collected papers of Srinivasa Ramanujan. Cambridge University Press, Cambridge, 1927.

4. Карацуба Е. А., Быстрое вычисление трансцендентных функций. Проблемы передачи информации (1991) 27, №4, 87-110.

5. Карацуба Е. А., Быстрое вычисление Г(3). Проблемы передачи информации (1993) 29, №1, 68-73.

6. Karatsuba Catherine A., Fast Evaluation of Bessel Functions. Integral Transforms and Special Functions (1993) 1, №4, 269-276.

7. Карацуба Е. А., Быстрое вычисление дзета-функции Римана $\zeta(s)$ для целых значений аргумента s. Проблемы передачи информации (1995) 31, №4, 69-80.

8. Карацуба Е. А., Быстрое вычисление дзета-функции Гурвица и $L$-рядов Дирихле. Проблемы передачи информачии (1998) 34, №4, 342-353.

9. Karatsuba Ekatherine A., Fast evaluation of hypergeometric function by FEE. Singapore: World Scientific. Ser. Approx. Decompos (1999) 11, 303-314.

10. Karatsuba E. A., On the computation of the Euler constant $\gamma$. J. Numer. Algor. (2000) 24, №1-2, 83-97.

11. Karatsuba E. A., Fast computation of $\zeta(3)$ and of some special integrals using the Ramanujan formula and polylogarithms. BIT Num. Math. (2001) 41, №4, 722-730.

12. Kummer E. E., Über die Convergenz und Divergenz der unendlichen Reihen. J. für die reine und angew. Math. (1835) 13, 171-183.

13. Kummer E. E., Eine neue Methode, die numerischen Summen langsam convergirender Reihen zu berechnen. J. für die reine und angew. Math. (1837) 16, 206-214.

14. Фихтенгольц Г. М., Курс дифференциального и интегрального исчисления (1959).

15. Bailey D. H., Borwein J. M., Mattingly A., Wightwick G., The computation of previously inaccessible digits of $\pi^{2}$ and Catalan's constant. Notices of $A M S$ (2013) 50, 844-854.

16. Catalan E., Memoire sur la transformation des series et sur quelques integrales defines. Mem. Acad. Roy. Belgique (1865) 33, 1-50.

17. Karacuba A., Berechnungen und die Kompliziertheit von Beziehungen. EIK (1975) 11, $10-12$.

18. Карацуба А. А., Сложность вычислений. Труды Математического института им. В.А. Стеклова (1995) 211, 169-183.

19. Карацуба А., Офман Ю., Умножение многозначных чисел на автоматах. Докладъ $А H$ CCCP (1962) 145, №2, 293-294. 
20. Тоом А.Л., О сложности схемы из функциональных элементов, реализующей умножение целых чисел. Доклады АН СССР (1963) 150, №2, 496-498.

21. Карацуба А. А., Комментарии к моим работам, написанные мной самим. В сб.: Совр. пробл. матем., 17. МИАН, Москва, 2013, с. 7-29.

22. Schönhage A., V. Strassen. Schnelle Multiplikation großer Zahlen. Comput. (1971) 7, 281292.

23. Fürer M., Faster integer multiplication. SIAM J. Comp. (2009) 39, №3, 979-1005.

Статья поступила 05.07.2013. 